\title{
Visual illusions with acute and obtuse angles: Configurational effects and observer strategies
}

\author{
PETER WENDEROTH and MICHAEL JOHNSON \\ University of Sydney, Sydney, N.S.W. 2006, Australia
}

\begin{abstract}
In three experiments, it is demonstrated that the negative Poggendorff illusions that Restle (1969) and others have reported are a special case, that, in general, acute- and obtuse-angle effects are the same, that there are additional special cases which modulate the illusion, but only slightly, and that these special cases derive from particular configurations which impose other effects, including observer strategies, on the basic illusion. These additional effects do not bear necessarily upon the fundamental explanation of the illusion. The experiments also show that effects obtained with amputated illusory figures are not linearly additive. Recent experiments that have measured apparent oblique separation in the Poggendorff figure and have found large obtuse-angle but small, or negative, acute-angle effects are also discussed. It is argued that these experiments have not demonstrated that the two dependent variables-alignment and length judgments-are correlated, or that one error causes the other; and it is argued that QuinaHolland's theory of parallel attraction to explain the illusion is not tenable.
\end{abstract}

During the course of more than a century's research on the Poggendorff illusion (see Robinson, 1972), numerous investigators have studied both the complete figure (Figure 1A) and its obtuse- and acuteangled amputations (Figures $1 \mathrm{~B}$ and $1 \mathrm{C}$, respectively). The general consensus has been that the apparent misalignment of the truly collinear obliques is roughly equivalent in the full figure and in the obtuse-angled versions, but that the effect is minimal with acute angles, or even reversed, such that the upper-right oblique in Figure 1C may appear not above but below the extension of the lower-left oblique (see Day, 1973; Green \& Hoyle, 1964; Imai, 1973; Judd, 1899; Krantz \& Weintraub, 1973; Restle, 1969; Weintraub \& Krantz, 1971; Wenderoth, 1980; Wenderoth \& Johnson, 1981).

Despite this evidence, the aim here is to demonstrate (1) that the acute-angle effect is a special case, (2) that, in general, acute- and obtuse-angle illusions are roughly equivalent, (3) that there are additional special cases in which illusions are unusually large or unusually small, and (4) that these special cases derive from particular configurations which impose other effects (or observer stratagems) on the Poggendorff illusion and hence do not bear necessarily upon its fundamental explanation.

Recently, it has been shown that negative errors occur with acute-angled figures when the oblique and parallel segments are equal or near equal in length (as, for example, in Restle's, 1969, experiment) but

This research was supported by an Australian Research Grants Committee award to the first author (Grant A 28015547 I) which included the second author's Research Assistantship. We thank the Committee and also the University of Sydney for provision of other facilities and minor consumables.
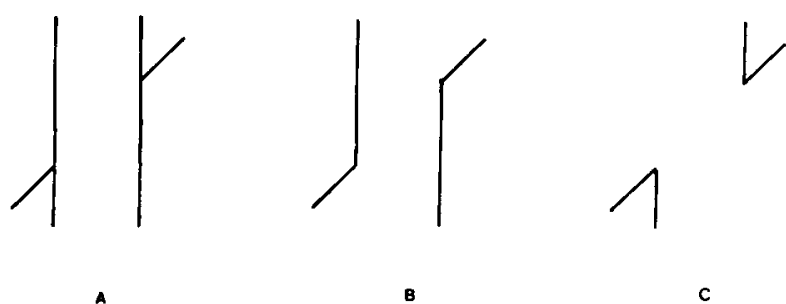

Figure 1. Variants of the Poggendorff illusion. (A) The full figure, in which the right oblique appears above the extension of the left oblique. (B) The obtuse-angled amputation, with similar effect. (C) The Restle, acute-angled figure which often shows a reversed (negative) effect.

that positive errors occur when the parallels are very much longer than the obliques (Day \& Dickinson, 1979; Wenderoth \& Johnson, 1981). One hypothesis to explain this difference is that the "gestalt" of the figure in the first case (Figure 1C) seduces observers into using a novel stratagem which they do not use with other configurations. That is, Figure $1 \mathrm{C}$ can be seen not only as a pair of oblique test lines intersecting a pair of vertical parallels but also as two oppositepointing acute angles. Seen in the latter mode, the right-hand angle in Figure IC appears to "point" below the left-hand angle, in that its virtual bisector passes below the vertex of the left-hand angle. Perhaps negative illusions are found with this display, because some subjects asked to align the obliques in fact adopt the stratagem of aligning the angle bisectors (Day, 1973; Wenderoth \& Johnson, 1981).

To test this hypothesis, albeit indirectly (see below), the general plan of the experiments to be described was systematically to vary the length of the vertical, parallel components of acute- and obtuse-angled am- 
putations. For example, in the first experiment, the parallels ranged from extremely short, much shorter than the oblique lines, to very long. Indeed, since the stimuli were generated on a visual display screen, the longest parallels used were off-screen, or continuous, such that no matter how low or high the right-hand oblique was set, any parallel attached to it always disappeared off-screen, behind a circular mask.

In all experiments, the oblique lines were $5 \mathrm{~mm}$ (.5 deg) long and the distance between the parallels was $20 \mathrm{~mm}(2 \mathrm{deg})$. The smallest parallels used over the three experiments were $1.2 \mathrm{~mm}$ long $(7-\mathrm{min} 14-$ sec arc). Sometimes the figures contained acute or obtuse angles only. On other occasions, the parallels were either extended beyond the intersect point by various amounts (overshoots) or stopped short of the intersect, leaving a gap (undershoots). Examples of these kinds of figures are shown in Figure 2. In every case, the observer's task was to adjust the height of the complete right-hand side (RHS) segment of the figure (oblique and parallel segment) so that the two oblique segments were aligned visually. The oblique lines were always oriented $45 \mathrm{deg}$. Errors in the usual Poggendorff direction (RHS set too low) were scored as positive; reverse errors were called negative.

Consider now two issues relating to the rationale of the experiments. First, if the small, or reversed,
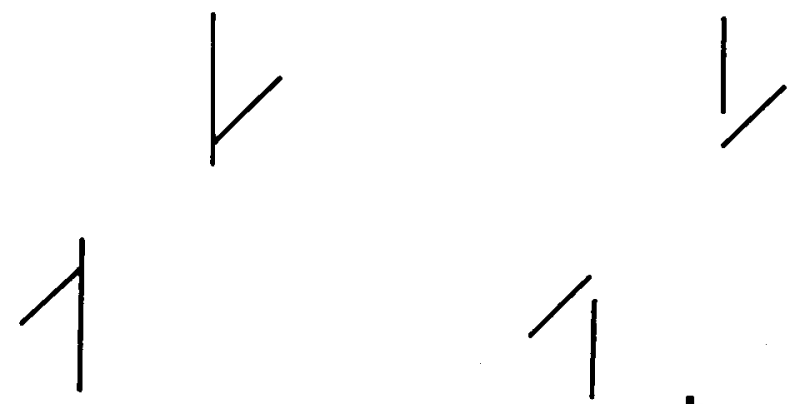

a

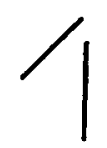

b
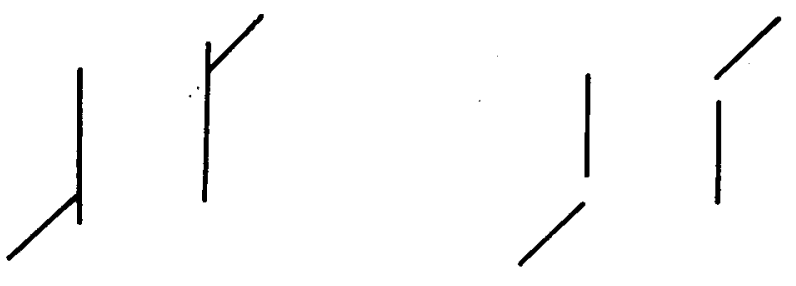

C

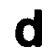

Figure 2. Examples of acute- and obtuse-angled amputations with parallel "overshoots" (a,c) and "undershoots" (b,d).

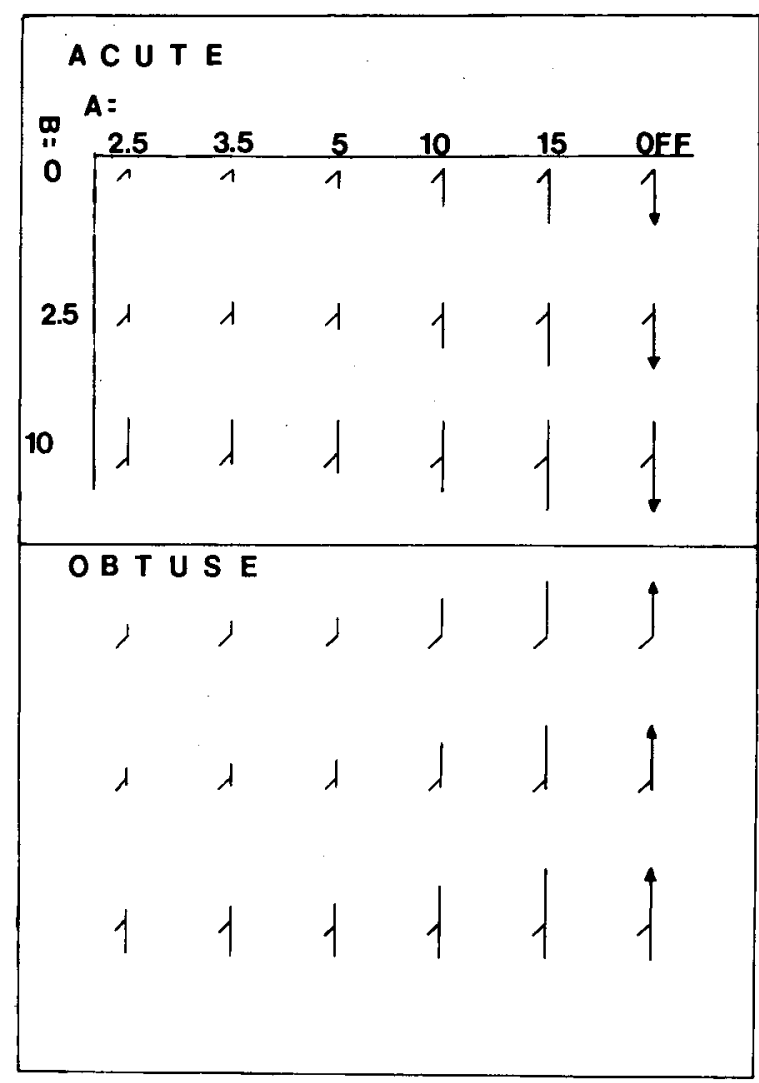

Figure 3. The stimulus set in Experiment 1, with oblique segment always $5 \mathrm{~mm}$ long. Left-hand side components only shown here, although all stimuli were dual sided, as in Figure 1. Upper panel: Acute angle figures. The length of the acute-angle arm varies from $A=2.5 \mathrm{~mm}$ through $3.5,5.0,10.0$, and $15.0 \mathrm{~mm}$, to offscreen (represented by arrowhead on arm). These parameters are identical for rows 2 and 3 except that, whereas there is no obtuseangle segment in row $1(B=0)$, there is one in rows 2 and 3 and its length is $2.5 \mathrm{~mm}$ (row 2) or $10.0 \mathrm{~mm}$ (row 3). Lower panel: As for upper panel, except all angles obtuse. Here, the column variable $(A)$ is obtuse-angle arm length; the row variable $(B)$ is the length of the tacked-on acute-angle.

acute-angle effect is a result of judging angle direction, why not test this directly, by asking subjects how they do the task, or by varying experimental instructions? As Green and Hoyle (1964) noted, quizzing observers results in variable responses and subjects are frequently unable clearly to report on how a task is performed. Instructing subjects to perform the task by using or not using the angle bisector strategy probably is not a useful approach: we have found that subjects instructed not to do so do not consistently produce positive or negative illusions, and there are many possible explanations for this finding. For example, the instruction might be irrelevant to the effect, or relevant but too difficult to follow, or simply not followed. At any rate, if the instruction to use the angle bisectors did produce negative effects, this would show merely that such instructions 
can mimic the usually obtained effect, not necessarily that such a stratagem is the sole explanation of it.

The second issue relating to rationale concerns why we believe that variation in parallel lengths might test, indirectly, the hypothesis that small, or negative, acute-angle effects occur because observers use anglebisector directions in making their judgments. Figure 3 shows all of the stimuli used in the first experiment [left-hand side (LHS) only is shown].

Parameter A is the length of the parallel, in millimeters, which varies from $2.5 \mathrm{~mm}$ to continuous (offscreen); parameter B is the length of any overshoot beyond the acute-angle vertex (upper panel) or obtuseangle vertex (lower panel). Thus, for example, the two stimuli that have no overshoot and have equallength obliques and parallels are those for which $A=5, B=0$.

The hypothesis is that when these lengths are equal, the angle bisector stratagem will dominate and negative illusions will occur. However, longer or shorter parallels, and overshoots, will tend to destroy the "angle pointing" configuration, so that subjects will tend to align the obliques, as instructed, and negative effects will be less likely to occur. Indeed, if positive illusions were obtained not only with very long but also with very short parallels, this would provide powerful evidence against any explanation of negative effects couched in terms either of acute angles in general or short parallel segments in general. Finally, negative effects are not predicted for obtuse angles when $A=5, B=0$ because the angle bisectors have directions totally removed from the alignment of the obliques, so that a confusion between oblique alignment and angle bisector alignment is virtually impossible.

\section{EXPERIMENT 1}

\section{Method}

Apparatus. Stimuli were presented on the flat face of a Tektronix 608 display (P4 phosphor) interfaced with a Hewlett-Packard graphics translator (H8-1350A) and a PDP-11/20 computer.

Subjects viewed the screen in a totally dark room from a distance of $57 \mathrm{~cm}$, with head upright on a padded chinrest. At this distance, $1 \mathrm{~cm}$ subtends 1 deg visual angle.

The display screen was masked by a black, circular cardboard mask, $100 \mathrm{~mm}$ in diameter. At low screen intensity levels, lines of different orientation differed noticeably in contrast. Hence, intensity was increased and neutral density filters (Polaroid sheets) were mounted over the aperture. In this way, all stimulus lines had an effective luminance of approximately $3 \mathrm{~cd} \mathrm{~m}^{-2}$ and contrast, defined as $\left(L_{\max }-L_{\min }\right) /\left(L_{\max }+L_{\min }\right)$, of approximately 1.0 .

Stimuli. The general stimulus dimensions have been given previously but are repeated here for clarity. All oblique test lines were $5 \mathrm{~mm}(.5 \mathrm{deg})$ long, and the width between parallels was always $20 \mathrm{~mm}(2.0 \mathrm{deg})$. There were a total of 36 stimuli (Figure 3), 18 called acute angles (upper panel) and 18 called obtuse angles (lower panel).

Each set of 18 figures was produced by a complete crossing of $6 \times 3$ parallel-segment lengths, as follows: In one set of six conditions (row labeled $B=0$ in the upper panel of Figure 3, and the corresponding row in the lower panel), the parallel length, for and acute- or obtuse-angle display simply increased from $2.5 \mathrm{~mm}$ (15 min arc) through $3.5 \mathrm{~mm}$ (21 $\mathrm{min} \mathrm{arc}), 5 \mathrm{~mm}$ (30 min $\mathrm{arc})$, $10 \mathrm{~mm}$ (60 $\mathrm{min}$ arc), $15 \mathrm{~mm}$ (90 min arc), to off-screen (continuous).

In another set of six conditions (rows $\mathrm{B}=2.5$ in Figure 3 ), all stimuli were as in $\mathrm{B}=0$ except that if the angles were acute, a 2.5-mm (15-min-arc) obtuse angle was tacked onto the acute angle; and if the $\mathrm{B}=0$ angles were obtuse, a $2.5-\mathrm{mm}$ acute angle was tacked on. In the third set of six stimuli (row $B=10.0$ in Figure 3), the same principle applied, but the tacked-on angle-arm lengths were $10 \mathrm{~mm}$ ( $60 \mathrm{~min}$ arc).

One additional condition was run: a control parallelless condition in which only the obliques were present. Prior to analysis, this mean error was subtracted from the other 36 means, for each subject individually.

As discussed earlier, the rationale (or one rationale) for the "tacked-on" segments was the hypothesis that if the Restle-type display does have the unique gestalt discussed earlier (the $A=5.0$, $B=0$ display in Figure 3), then tacking on a small obtuse angle might destroy this gestalt (as, for example, in $\mathrm{A}=5.0, \mathrm{~B}=2.5$ or 10.0 in Figure 3) and thereby eradicate negative illusions.

Procedure. Each subject made two settings of the RHS of each of the 36 stimuli to produce visual collinearity, from starting positions that were chosen randomly but were restricted to being within $20 \mathrm{~mm}$ of objective equality. Three microswitches were mounted before the subject. A press on the center switch initiated or terminated a trial; and presses on the outer switches moved the RHS up or down at an average rate of $2-3 \mathrm{deg} / \mathrm{sec}$ (the slight variation occurred because in the user-sharing system on the PDP-11 number of users active affects this parameter; however, variation was slight and did not seem to affect settings markedly). Each subject completed the 36 conditions in a different random order, although the paired settings for each condition were consecutive.

Subjects. There were 18 subjects, 6 males and 12 females, all with emmetropic or corrected vision, who were volunteers from an introductory psychology course. Except for a 22-year-old female and a 28 -year-old male, all were aged under 21 .

\section{Results}

The mean errors obtained as a function of anglearm length are shown in Figure 4 for both acute (Figure 4A) and obtuse (Figure 4B) angles.

These mean errors are all experimental minus control means (the overall mean parallelless illusion was $2.42 \mathrm{~mm}$, with standard error .48).

As Figure 4A shows, the sole negative illusion occurred in the acute-angle case in which the parallel length equalled the oblique length $(A=5.0, B=0)$. This is the Restle stimulus and the result that was predicted. Shorter or longer parallels produced positive illusions. No negative, or even near-negative, illusions occurred for the $A=5.0$ conditions when small obtuse angles also were attached. Indeed, the most general description of Figure 4, perhaps, is that the largest effects of all seemed to occur when there was an obtuse-angle arm length of 5.0 or $10.0 \mathrm{~mm}$, together with an acute-angle arm length of about 2.5 or $5.0 \mathrm{~mm}$. It is beyond the scope of this experiment to speculate about the role of relative acute- and obtuse-angle arm lengths in the illusion, essentially because only a small sample of length values (especially for B) was used. However, it might be profitable to examine a greater range of values in further experiments because, in Figure 4, the largest ob- 


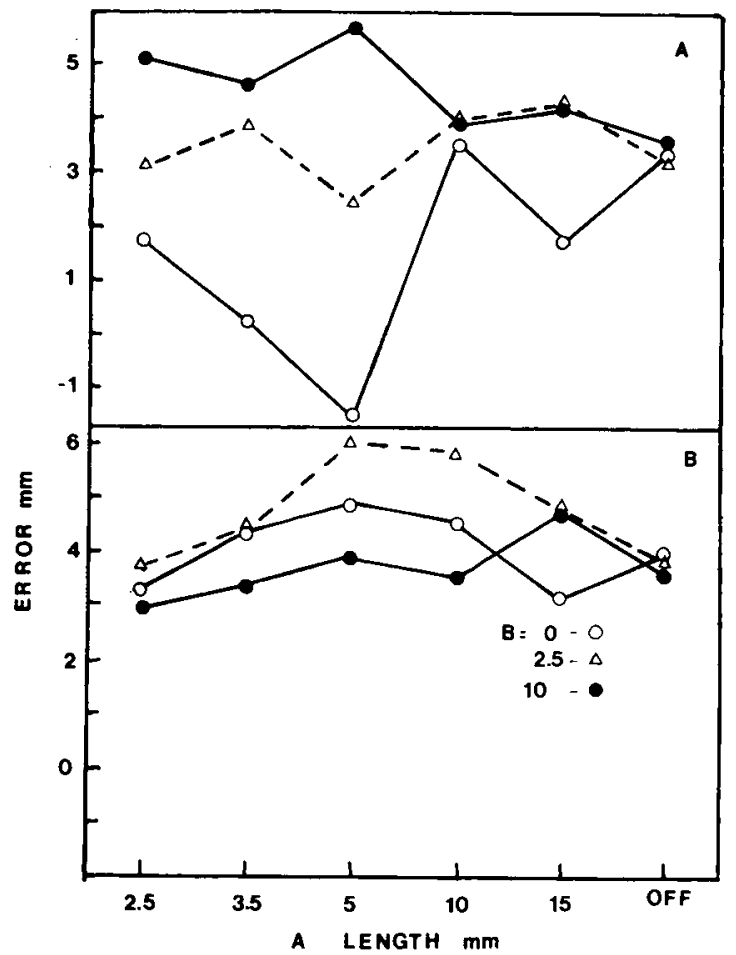

Figure 4. Mean alignment errors in Experiment 1. Upper panel: Errors (mm) as a function of acute-angle arm length $(A)$ for values of $B=0$ (open circles), $2.5 \mathrm{~mm}$ (open triangles), and $10.0 \mathrm{~mm}$ (filled circles). Lower panel: As for upper panel, for obtuse-angle set (refer to Figure 3).

tained effects consistently occurred when the acute/ obtuse parallel ratio was $.25, .35$, and .50 (Figure $4 \mathrm{~A}$; three filled circles at upper left) and .25 and .50 (Figure 4B; two open triangles at upper center).

Statistically, planned orthogonal contrasts based on a simple subjects $\times$ treatments design (Winer, 1962, chap. 4) characterized the data as follows. For each of the six functions in Figure 4, tests of trend across values of $A$ were carried out (30 contrasts). ${ }^{1}$ Next, for acute angles (Figure 4A), one contrast tested for an overall mean difference between $B=0$ and $B=10.0$ and another contrast asked whether $B=2.5$ was equal to the average of the two former conditions. Two identical contrasts were applied to the obtuse-angle data. Finally, the 35th contrast tested the overall acute-angle mean and obtuse-angle mean difference.

Beginning with the last of these, the overall obtuseangle illusion $(+4.19 \mathrm{~mm})$ exceeded the acute-angle mean $(+3.18 \mathrm{~mm})[\mathrm{F}(1,595)=37.10, \mathrm{p}<.001]$.

For acute angles (Figure $4 \mathrm{~A}$ ), the $\mathrm{B}=10.0$ mean $(+4.54)$ exceeded the $B=0$ mean $(+1.50)(F=112.39$, $\mathrm{p}<.001)$ and the $\mathrm{B}=2.5$ mean $(+3.51)$ did exceed the average of the other two $(+3.02)$, but not markedly $(\mathrm{F}=3.95, \mathrm{p}<.05)$.

In the obtuse conditions (Figure $4 B$ ), the $B=0$ and $B=10.0$ means were not different $(+4.05$ and
$+3.74 \mathrm{~mm}$, respectively; $\mathrm{F}=1.14, \mathrm{p}>.05)$. However, the $B=2.5$ mean $(+4.79)$ was larger, not only than the average of the other two $(+3.90 \mathrm{~mm} ; \mathrm{F}=$ $12.91, \mathrm{p}<.001$ ), but also than each of them.

The tests of trend over the individual functions describe them aptly. Quadratic trend components were significant for only the acute, $\mathrm{B}=0$ function, because it dips to negative near the center $(F=11.66$, $\mathrm{p}<.001$ ) and for the obtuse, $\mathrm{B}=2.5$ function, because it peaks near the center $(F=16.56, p<.001)$. Also significant, for the acute, $B=0$ function, were linear $(F=20.17)$ and cubic $(F=12.95)$ trends, with $\mathrm{p}<.001$ in each case. These reflect, respectively, the larger effects for large $A$ values and flattening off at larger values of $\mathbf{A}$.

Linear trend was significant in only two other cases: first, for acute, $B=10.0(F=5.87, p<.05)$, reflecting the negative slope of the function; and second, for obtuse, $B=10.0(F=6.67, p<.01)$, here reflecting the positive slope. The irregularity of the acute, $B=10.0$ curve sufficed to produce significant quintic trend as well $(\mathrm{F}=4.71, \mathrm{p}<.05)$.

Finally, both the acute $B=2.5$ and obtuse $B=0$ functions were relatively flat but irregular: hence, in the first case only the quartic trend was significant $(F=4.13, p<.05)$; in the latter, it was the cubic trend $(\mathrm{F}=3.92, \mathrm{p}<.05) .^{2}$

\section{Discussion}

The result of major interest in Experiment 1 was that the sole negative illusion occurred with a Restletype display, when the parallel arms of the acuteangled amputation were equal in length to the oblique segments. Indeed, when the parallel arms were very short $(2.5 \mathrm{~mm}$ or half the oblique length), the experimental minus control illusion was $+1.72 \mathrm{~mm}$, almost three standard errors $(.64 \mathrm{~mm})$ greater than zero; and when the parallel arms were infinitely long (off-screen), acute-angled amputations ( $A=$ offscreen, $B=0$ ) resulted in illusions that were clearly positive $(+3.40 \mathrm{~mm}$; $\mathrm{SE}, .66)$ and similar in magnitude to obtuse-angle effects $(A=$ off-screen, $B=0$; $+4.01 \mathrm{~mm}$; SE, .65).

Although the result is consistent with the hypothesis outlined earlier, concerning the special "gestalt" of Figure $1 \mathrm{C}$, it is conceivable that the illusions obtained when very short parallel segments are used involve another confounding factor. Consider Figure 5, remembering that the usual Poggendorff effect is such that the upper right oblique appears too high and hence is set too low when apparently aligned.

At least two factors could affect judgments of alignment when acute- or obtuse-angle parallels are extremely short, as in Figure 5.

First, the illusion literature is replete with examples of stimuli (lines, dots, circles, etc.) which show "contrast" effects when moderately separated but "assimilation" effects when very close (see Robinson, 


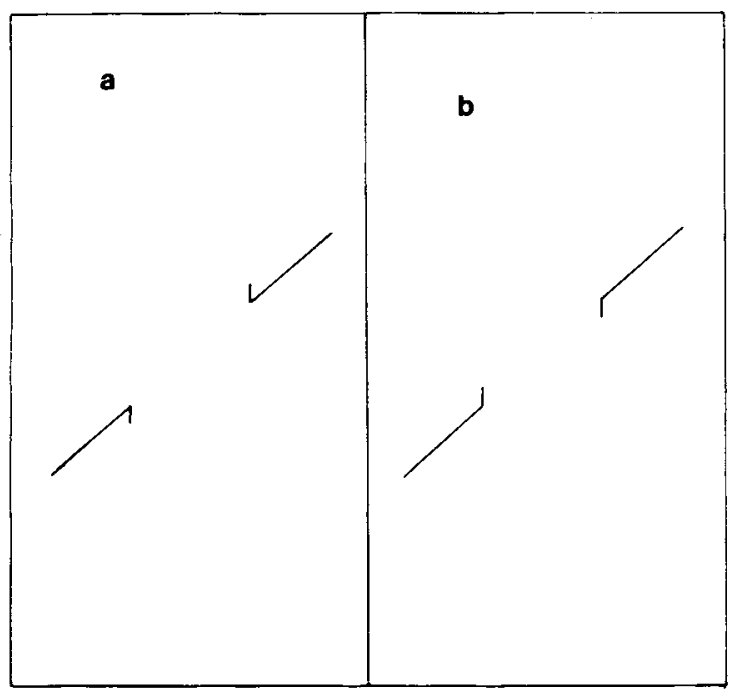

Figure 5. Amputations of the Poggendorff illusion with very short acute-angled parallels (a) or obtuse-angled parallels (b).

1972). Suppose that the obliques in stimuli such as those in Figure 5 perceptually assimilated towards the tips of the very short parallel arms. In that event, the obtuse angle effect would be reduced: the upperright oblique would appear lower and so be set higher. Similarly, the acute angle effect would be increased.

Such a process could indeed explain why large illusions tended to occur in Experiment 1 when obtuseangled stimuli had small acute overshoots, but not when acute-angled stimuli had small obtuse overshoots. In the former case, the illusion, assuming additivity, would be given by: obtuse effect plus acute assimilation. In the latter case, it would be given by: acute effect minus obtuse assimilation.

The second factor that could affect judgments of such short-arm stimuli relates not to perceptual mechanisms but to observer strategies or assumptions. When the distance between the obliques and parallel tips is extremely short, observers might simply assume, or guess, that the correct setting is to position the oblique at the tip.

Fortunately, a simple test can be devised to discriminate between these two possible factors because they predict opposite outcomes: deliberately setting the oblique towards the short parallel tip would reduce alignment errors in obtuse-angled amputations with very small acute overshoots and would increase errors in acute amputations with very small obtuse overshoots. Experiment 2 was designed to test these hypotheses.

\section{EXPERIMENT 2}

The rationale of this experiment was as follows. First, if "assimilation" effects occurred, then obtuseangle figures with very small acute overshoots would induce larger effects than acute-angle figures with small obtuse overshoots. Second, if subjects adopted the stratagem of setting the oblique to the short parallel tip, not only would the reverse differences occur, but also identical, but opposite, differences should occur in any undershoot (gap) conditions: acute-angle effects should be enhanced; obtuse-angle effects should be reduced (see Figures $2 b$ and $2 d$ ).

\section{Method}

In this experiment, acute-angled and obtuse-angled amputations always had off-screen parallel arms. However, at the angle vertex, there was either a tacked-on, small obtuse (acute) angle of variable length or a gap of variable length (as in Figure 2). These "overshoots" or "undershoots" varied in length from $+2.4 \mathrm{~mm}$ (14 min $28 \mathrm{sec}$ arc) maximum overshoot, through zero, to $-2.4 \mathrm{~mm}$ undershoot (gap) in equal steps of .4 mm ( $2 \mathrm{~min} 25 \mathrm{sec}$ arc). Thus, each subject completed two settings under 13 undershoot or overshoot conditions (six of each plus zero) for both acute and obtuse angles, giving 26 conditions in all. In all other respects, the methods and procedures were as in Experiment 1 . Fifteen subjects, 5 males and 10 females, were used. All were under 21 except for one female admitting to "over $\mathbf{4 0}$."

\section{Results}

The mean illusions obtained are shown in Figure 6.

Inspection of the data is sufficient to show that small overshoots did result in the predicted assimilation effects.

Although there appeared to be a tendency for much smaller reverse effects to occur with gaps, these were not significant: 13 planned contrasts between each pair of means in Figure 6, using the same statistical design as in Experiment 1, showed that only three mean differences between acute and obtuse angles were significant. These occurred when there were overshoots of $1.2,1.6$, and $2.0 \mathrm{~mm}[\mathrm{Fs}(1,340)=$ $7.32,10.69$, and 4.58 , ps $<.01,<.01$, and $<.05$, respectively].

Other contrasts indicated that the average of the acute- and obtuse-angle functions increased linearly $(F=30.87, p<.001)$, with no significant residual trend $[F(11,350)=1.69, p>.05]$.

\section{Discussion}

Figure 6 not only shows that the predicted "assimilation" effects occurred with overshoots, and not

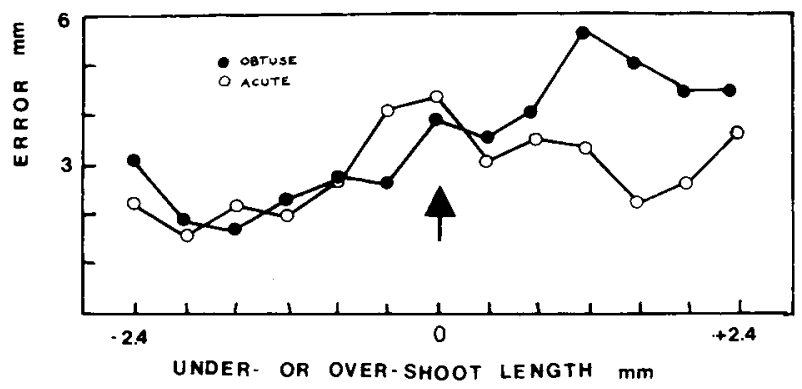

Figure 6. Mean alignment errors, Experiment 2, as a function of undershoot (gap) or overshoot length in acute-angled figures (open circles) or obtuse-angled figures (filled circles). 
with undershoots, but also suggests that these assimilations occur only with moderately short line segments: the mean differences when the overshoots were significant between 1.2 and $2.0 \mathrm{~mm}$, but not when the overshoots were smaller $(.4$ to $.8 \mathrm{~mm})$ or longer $(2.4 \mathrm{~mm})$.

Unfortunately, the range of overshoots used was not sufficient to indicate whether the two functions in Figure 6 merely converge, and remain so, for overshoots of $2.4 \mathrm{~mm}$ or longer: the evidence for convergence is based on only one nonsignificant difference, at $2.4 \mathrm{~mm}$. In a final experiment, therefore, we varied the overshoot from 0 to $7.2 \mathrm{~mm}$, in seven 1.2-mm steps. In addition, the experiment included stimuli with and without off-screen angle arms, the latter conditions thus providing replications of the effects measured in Experiment 1 but with a greater range of stimuli. For example, with acute (or obtuse) angle obliques $5 \mathrm{~mm}$ long, the parallel segments varied from 0 to $7.2 \mathrm{~mm}$ in $1.2-\mathrm{mm}$ steps. This included no conditions in which the parallels equaled the obliques, but did include three shorter-than and three longer-than conditions.

\section{EXPERIMENT 3}

\section{Method}

There were 28 stimulus displays in Experiment 3, 14 called "acute" and 14 called "obtuse." Consider the "acute" conditions. Half (seven) of the figures had off-screen, vertical acute parallels, as in Figure 3, $A=$ OFF conditions. The variable over these seven stimuli was the length of the tacked-on obtuse-angle. This varied from 0 (Figure $3, A=O F F, B=0$ ), through 1.2, 2.4, $3.6,4.8$, and $6.0 \mathrm{~mm}$, to $7.2 \mathrm{~mm}$. The other seven "acute" stimuli were identical except that the off-screen segment was absent. So this second set of "acute" stimuli were in fact all small obtuse angles: they consisted of the tacked-on parallel and the oblique only.

The 14 "obtuse" stimuli were similarly derived. Seven had off-screen obtuse-angled parallels, with tacked-on acute angles from 0 to $7.2 \mathrm{~mm}$ long. The other seven stimuli were the same, but lacked the off-screen segment and so were, in effect, small acute angles.

Methods and techniques were exactly as in the other experiments. There were 15 subjects, 3 males and 12 females, from an introductory psychology course. All were under 21 except for one 25-year-old female.

\section{Results}

The mean alignment errors obtained are shown in Figure 7. To avoid confusion between stimuli that were called "acute" but were actually small obtuse angles (and vice versa), schematic stimuli have been inserted to the right of each of the four functions in Figure 7.

In the lower panel of Figure 7, note first that small acute angles (i.e., obtuse angles with off-screen arm missing) virtually replicated the results of Experiment 1 . Errors were positive when the acute parallels were very short $(1.2$ or $2.4 \mathrm{~mm})$ but negative when

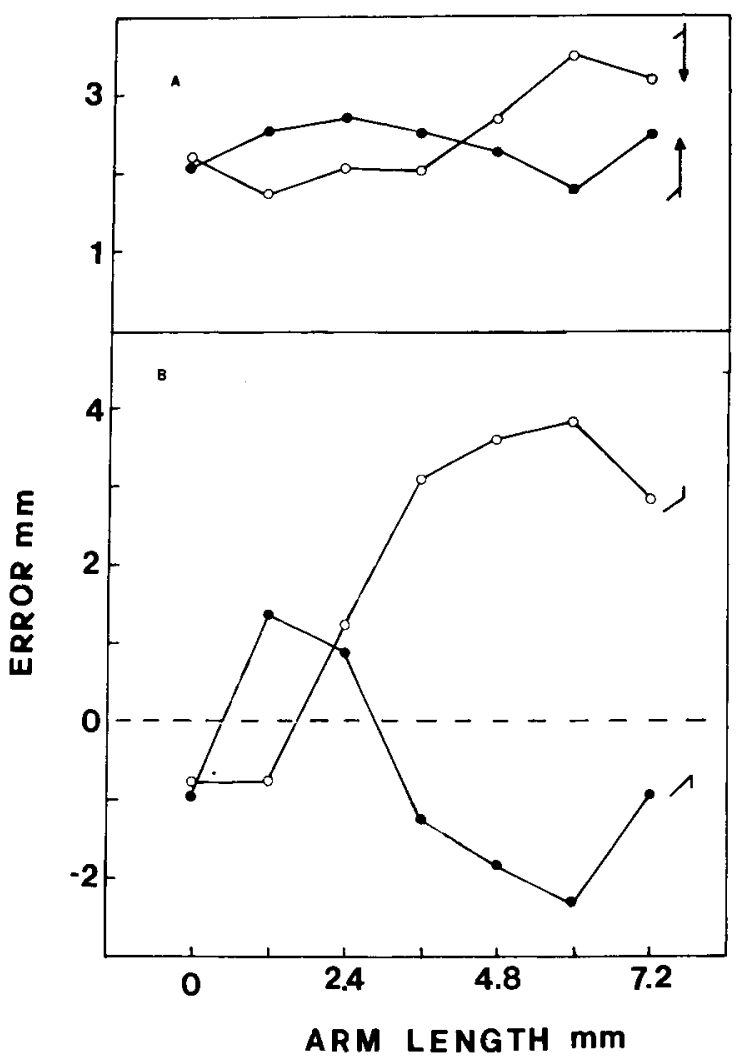

Figure 7. Mean alignment errors, Experiment 3. Upper panel: Alignment errors as a function of length of parallel-arm overshoot for acute- (open circles) and obtuse- (filled circles) angles with off-screen parallel angle arms. Lower panel: As for upper panel except that off-screen arms are omitted. (Note that an acute angle with overshoot and of fscreen arm missing actually is a small obtuseangle, and vice versa.)

the parallel length approximated the $5-\mathrm{mm}$ oblique length (at 4.8 and $6.0 \mathrm{~mm}$ ). ${ }^{3}$ In the case of small obtuse angles (i.e., acute angles with off-screen arm missing), no such reversals occurred: errors merely increased almost monotonically with obtuse-arm length.

How important are these configurational effects when off-screen arms are present? The upper panel shows that when the extra arm length (overshoot) is zero, the errors obtained with off-screen arms, whether acute or obtuse, are virtually identical $(+2.22$ and $+2.09 \mathrm{~mm}$, respectively). It might be noted that these errors are small relative to those usually obtained-some subjects gave very small effects-but that is not relevant here. Rather, the relative effect sizes are of interest. As the overshoot increases to $2.4 \mathrm{~mm}$, the acute- and obtuse-angle functions appear to separate, precisely as they did in Experiment 2 (Figure 6). Presumably, this represents the "assimilation" effect. Then, as Experiment 2 foreshadowed, the functions converge again and even appear to reverse. 
However, statistical tests, again using planned contrasts and the same statistical design as before, showed that of the 14 mean pair differences, 7 in the upper panel of Figure 7 and 7 in the lower panel, only 4 were significant. None of the mean pairs in the upper panel differed (no doubt an overall test of interaction would be significant, but the planned contrasts analysis was selected, of course, beforehand). In the lower panel, only the four right-hand means were different, for arm lengths 3.6, 4.8,6.0, and $7.2 \mathrm{~mm}[\mathrm{~F}(1,378)=$ $141.92,222.20,282.10$, and 105.86 , respectively, $\mathrm{p}<.0001$ in each case].

The combination (average) of the upper-panel function showed no significant trend at all. The average lower-panel function showed a significant linear increase only $(F=4.23, p<.05)$. The apparent quadratic trend was not significant $(F=2.95, p>.05)$, nor were any higher order trends.

Last, the overall mean error with off-screen parallels (mean of upper panel: $+2.40 \mathrm{~mm}$ ) was greater than the overall lower-panel mean $(+.58 \mathrm{~mm})$ when off-screen parallels were absent $(F=35.37, p<.0001)$.

\section{Discussion}

This experiment replicated the findings of Experiment 1 in demonstrating the unique case of the Restletype stimulus, in which negative effects occur only when an acute-angled amputation has equal, or nearequal, oblique and parallel arms. The experiment also replicated the "assimilation" effects of Experiment 2, which occur with small parallel overshoots, although none of the mean-pair contrasts was significant.

The most general summary of the results is that when parallel arms are long (off-screen), acute- and obtuse-angle illusions are the same (left-hand points, upper panel, Figure 7). Restle-type effects, or assimilation effects, which do occur under certain conditions, either are not relevant to long-parallel effects or merely cause ripples on the data surface, as the fluctuations across the upper panel of Figure 7 show. Here, the mean error is $2.40 \mathrm{~mm}$, and the range of errors is only 1.72 to $3.19 \mathrm{~mm}$.

\section{GENERAL DISCUSSION}

The general tenor of these experiments is, in a sense, negative, in that they demonstrate conclusively that certain avenues of experiment should not be followed if the Poggendorff illusion is to be explained. The experiments also make the point quite strongly that it is not empirically justifiable to make the assumption that additive components of the full illusion can be isolated by studying amputated figures. This point is most visible in Experiment 3: as Figure 7 shows, when the off-screen arms are absent, the acute and obtuse-angle effects, with $6.0-\mathrm{mm}$ parallel arm lengths, differ by +3.83 minus $-2.30 \mathrm{~mm}$, or
$6.13 \mathrm{~mm}$. However, when the stimuli are identical except for the addition of the off-screen arms, the difference is +3.25 minus +1.78 , or $1.47 \mathrm{~mm}$. Thus, off-screen acute- and obtuse-angle arms alone (no overshoot) induce the same effect; overshoots alone (small-arm acute or obtuse angles) induce large obtuseand acute-angle differences; but the difference between the combination stimuli is not large.

We continue to work at finding the mechanism of Poggendorff effects with long parallels: the classic effect. These experiments show that numerous special cases of amputated figures may give odd or unusual results, but that they are unlikely to bear upon the basic explanation of the complete effect. A similar point has been made recently in relation to another set of cases of unusual Poggendorff-like figures (Wenderoth, 1981), which may not bear upon the basic, original illusion.

A final issue that is relevant here also relates to theories of the Poggendorff effect. Two experimental groups recently have attributed the Poggendorff illusion obtained with the full figure and its amputations to an underestimation of the distance between the inner tips of the obliques (Greist-Bousquet \& Schiffman, 1981; Quina-Holland, 1977), following the earlier suggestion by Zanuttini (1976) that the parallels attract, or assimilate, to produce the apparent misalignment. Quina-Holland proposes that parallel attraction explains the illusion, whereas GreistBousquet and Schiffman prefer to explain it in terms of another illusion, the Mueller-Lyer. These studies are directly relevant here because both obtained large effects with obtuse-angled amputations but not with acute-angled figures.

The first, general, point to be made is that neither study obtained classic oblique alignment judgments as well, so that it is not known whether the distance underestimates correlated with the usual Poggendorff effects. In both studies, such correlation was inferred from the fact that small or reverse effects occurred with Restle-like acute-angle figures. However, even if we allow for the absence of such data, it could only be concluded, at best, that there is a correlation between one way of judging a figure and another. Imagine if, 100 years ago, it had been discovered that the interoblique distance appeared smaller. Would a recent discovery of apparent misalignment of the obliques then be taken to explain the effect? Certainly not: the two effects merely covary.

The second, general, point is that there is a real need to correlate the distance and alignment judgments because there are many instances in perceptual research in which euclidean relations do not obtain in perceptual judgments, due to observers' using different strategies in making different judgments on the same figure. For example, one acute angle may show greater arm repulsion than another (parallel matching) but simultaneously be judged a smaller 
angle (Wenderoth \& White, 1979). Indeed, in some sense, one could argue here that if the upper-right Poggendorff oblique looks higher than it is, then the interoblique distance should look longer than it is, in simplistic euclidean terms. The point is that there is no guarantee that the distance and alignment judgmental errors will correlate.

Finally, some specific points relate to QuinaHolland's (1977) own data, which seem convincingly to falsify her own hypothesis. First, when she varied the angle of the obliques, Quina-Holland frequently obtained distance overestimations (i.e., reversals) for near-90-deg angles. There is no evidence that negative alignment errors occur under such circumstances (e.g., Anton, 1976; Hotopf, Ollerearnshaw, \& Brown, 1974). Second, she obtained 10\%-20\% overestimations for 90 -deg angles, which do not produce a large, if any, Poggendorff effect, let alone a negative effect. Yet, instead of drawing the plausible inference that the alignment and distance effects are not correlated, Quina-Holland actually asserts that her data "reveal serious shortcomings of previous methods of describing the illusion, and indicate that the distance factor should be considered in future evaluations of the Poggendorff illusion"' (p. 122). It can also be pointed out that Quina and Pollack (1973) obtained nonmonotonic parallel attraction as a function of line separation, whereas the Poggendorff effect not only increases monotonically with separation (Wenderoth, Beh, \& White, 1978), but indeed, virtually is unaffected by the removal of the second parallel and its replacement with a single dot (e.g., Wenderoth \& Johnson, 1981). Is the oblique-to-dot distance in that sort of configuration significantly underestimated? Lastly, it is relevant to note that Wenderoth and Johnson (1981) obtained larger alignment illusions in figures with both LHS and RHS obtuse amputations than in figures with one acute, one obtuse. Yet, in the former case, the parallels are vertically separated; in the latter, they are opposite.

It seems justified, then, to conclude at this stage that sufficient data still are not available regarding which key variables affect alignment judgments. Hopefully, experiments such as those reported here will eventually clarify the role of all relevant factors. Before this is achieved, it seems imprudent to study new dependent variables in the absence of alignment judgments lest these prove, in the long term, to be mere epiphenomena.

\section{REFERENCES}

Anton, B. S. Poggendorff illusion as a function of orientation of transversal and parallel lines. Perceptual and Motor Skills, $1976,43,83-90$.

DAY, R. H. The Poggendorff illusion with obtuse and acute angles. Perception \& Psychophysics, 1973, 14, 590-596.

DAY, R. H., \& Dickinson, R. G. Relative magnitude of apparent misalignment in acute-angle and oblique-line figures. Perception \& Psychophysics, 1979, 25, 244-246.

Green, R. T., \& Hoyle, E. M. The influence of spatial orientation on the Poggendorff illusion. Acta Psychologica, 1964, 22, 348-366.

Greist-Bousquet, S., \& Schiffman, H. R. The Poggendorff illusion: An illusion of linear extent? Perception, 1981, 10, 155-164.

Hotopf, W. H. N., Ollerearnshaw, C., \& Brown, S. The regression to right-angles tendency and the Poggendorff illusion. III. British Journal of Psychology, 1974, 65, 213-231.

Imal, S. Experiments on Poggendorff illusion. Journal of Social Sciences and Humanities of Tokyo Metropolitan University, 1973, 2, 1-39.

Judn, C. H. A study of geometrical illusions. Psychological Review, $1899,6,241-261$.

Krantz, D. H., \& Weintraub, D. J. Factors affecting perceived orientation of the Poggendorff transversal. Perception \& Psychophysics, 1973, 14, 511-517.

Quina, K., \& Pollack, R. H. Attraction of parallels as a function of intercontour distance. Perceptual and Motor Skills, 1973, 36, 934.

Quina-Holland, K. Spatial distortions within the Poggendorff figure and its variants: A parametric analysis. Perception \& Psychophysics, 1977, 21, 118-124.

RESTLE, F. Illusions of a bent line. Perception \& Psychophysics, 1969, 5, 273-274.

Roвinson, J. O. The psychology of visual illusion. London: Hutchinson, 1972.

Weintraub, D. J., \& Krantz, D. H. The Poggendorff illusion: Amputations, rotations and other perturbations. Perception \& Psychophysics, 1971, 10, 257-264.

WENDERoth, P. A theoretical puzzle in a visual alignment task. Australian Journal of Psychology, 1980, 32, 45-52.

Wende roth, P. Bending the Poggendorff parallels and the rules of inference: A note on Brigell and Uhlarik. Perception \& Psychophysics, 1981, 29, 283-284.

Wenderoth, P., BEH, H., \& White, D. Alignment errors to both ends of acute- and obtuse-angle arms. Perception \& Psychophysics, 1978, 23, 475-482.

Wenderoth, P., \& Johnson, M. Are the obtuse-angles the key components of the Poggendorff illusion? Perception, 1981, 10, 165-172.

Wenderoth, P., \& White, D. Angle matching illusions and perceived line orientation. Perception, 1979, 8, 565-575.

Winer, B. J. Statistical principles in experimental design. New York: McGraw-Hill, 1962.

Zanutrini, L. A new explanation for the Poggendorff illusion. Perception \& Psychophysics, 1976, 20, 29-32.

\section{NOTES}

1. Tests of trend used standard contrast coefficients for equally spaced independent-variable values. Hence, these values are plotted with equal spacing in Figure 3, and the trends refer to functions plotted on such coordinates.

2. There were three cases in which stimuli in the acute-angle set and obtuse-angle set were identical. These were, for acute and obtuse conditions, respectively: (1) (A 2.5, B 2.5) and (A 2.5, B 2.5); (2) (A 10.0, B 10.0) and (A 10.0, B 20.0); and (3) (A 10.0, B 2.5) and (A 2.5, B 10.0). The obtained mean pairs were: 3.11 and $3.74 \mathrm{~mm}, 3.92$ and $3.86 \mathrm{~mm}$, and 4.02 and $2.94 \mathrm{~mm}$. This seemed, overall, to be a reasonable degree of agreement.

3. For the arm length $=0$ condition, there are no parallels at all. The obtained error is slightly negative because this same condition was run, as always, as a control before the experiment began. All means here are experimental minus control differences.

(Manuscript received May 21, 1981; revision accepted for publication October 30, 1981.) 